\title{
Presentazione all'editoriale del Dottor Riccardo Magistroni
}

Negli ultimi vent'anni del Novecento chi ha assistito dei pazienti affetti da malattia policistica (ADPKD) sa di aver "recitato" una parte prevalentemente passiva, poco più di una comparsa. Ricordo bene che essere al cospetto di un paziente con ADPKD, in modo più o meno palese, era come aprire le braccia in segno di sconforto e rassegnazione per la nostra impotenza a frenare l'evoluzione della malattia di cui il paziente in primis, e tutti noi a seguire, eravamo in balia. Potevamo impegnarci solo per contenere al meglio la malattia trattando l'ipertensione e altri fattori che sapevamo essere di secondaria importanza e comunque non risolutivi dello scenario di una patologia così importante per i nostri pazienti e le loro famiglie. Una malattia tanto devastante per tutti proprio per la mancanza di un qualsiasi tool terapeutico efficace.

Con l'inizio del nuovo secolo è stato evidente che esisteva una luce in fondo al tunnel dell'ADPKD, che si intravedeva anche solo di riflesso. Oggi, quasi vent'anni dopo, le cose sono effettivamente cambiate. Seppur ancora lontana, la luce della fine del tunnel è chiaramente visibile e solo una certa distanza ci separa da quel punto aperto. Le speranze per i pazienti, per le loro famiglie e anche per noi operatori sanitari, di poter svolgere un ruolo più proattivo stanno diventando una certezza. Gli studi e le ricerche in questo senso non sono più solo episodici e marginali come nel secolo scorso ma sono progressivamente sempre più importanti, sempre più incalzanti quasi a tamburo battente. I nefrologi e i ricercatori di altre branche che vi si dedicano sono sempre più determinati e indirizzati a sconfiggere questa patologia e i risultati non tarderanno a venire anche se ancora un po' di pazienza è richiesta... ma è d'obbligo, le cose devono essere fatte nel modo più scientifico e corretto. La scienza (prevalentemente nefrologica) intorno a questa patologia è organizzata, il paziente non è più solo ci sono associazioni, onlus e società scientifiche che si impegnano in ogni senso.

Per questo il Giornale apre con un editoriale di un oramai importante ricercatore italiano in questo campo. Ne abbiamo diversi a onor del vero, ma il Dott. Riccardo Magistroni mi ha di recente proposto una revisione dello studio su tolvaptan, una molecola che molti di noi hanno conosciuto e usato per le sindromi da inappropriata secrezione da ADH e che sta conoscendo una seconda... ma che dire una prima giovinezza nel suo possibile uso nell'ADPKD.

In questa lucida, seppur non semplice, dissertazione sullo studio di fase III sul tolvaptan e il suo studio di estensione (studio TEMPO 3:4 e TEMPO 4:4) il paziente potrà per l'appunto capire le doverose difficoltà che accompagnano la ricerca in questo campo ma anche che il movimento in questa direzione è in atto, certo, robusto e convincente e che pertanto la speranza di sbucare dal tunnel della malattia alla luce del sole è oramai solo questione di tempo, e non credo poi molto ... si va avanti!

\section{Marco Lombardi}

Editor in Chief

Giornale di Tecniche Nefrologiche e Dialitiche, Firenze
Luisa Sternfeld Pavia

Presidente AIRP, Milano 